Conclusions The CFA analyses indicate that while the fit of the four-factor model for the PDQ fits, the model could be improved. Particularly concerning is the different factor-pathways for seven items, item 19's current low item-factor reliability, and the increased correlations between factors. In adult SLE patients, researchers and clinicians should be cautious in interpreting PDQ-20 results using the current four factors (subscales). Further validity analyses, including exploratory factor analyses, are needed.

\section{CS-23 REMISSION AND LOW DISEASE ACTIVITY STATE ARE ASSOCIATED WITH A BETTER HEALTH-RELATED QUALITY OF LIFE IN SYSTEMIC LUPUS ERYTHEMATOSUS IN A PRIMARILY MESTIZO POPULATION}

\footnotetext{
${ }^{1,2}$ Manuel F Ugarte-Gil ${ }^{*}$, ${ }^{1}$ Rocio V Gamboa-Cardenas, 'Mariela Medina-Chinchón, ${ }^{1}$ Francisco Zevallos, ${ }^{1}$ Cristina Reátegui-Sokolova, ${ }^{1,2}$ Claudia Elera-Fitzcarrald, ${ }^{1}$ Victor Pimentel-Quiroz, ${ }^{1}$ Jose Alfaro-Lozano, 1,3Zoila Rodriguez-Bellido, ${ }^{1,3}$ Cesar A PastorAsurza, ${ }^{4}$ Graciela S Alarcón, ${ }^{1,3}$ Risto Perich-Campos. ${ }^{1}$ Rheumatology Department. Hospital Guillermo Almenara Irigoyen. EsSalud; ${ }^{2}$ Universidad Cientifica del Sur; ${ }^{3}$ Universidad Nacional Mayor de San Marcos; ${ }^{4}$ School of Medicine, University of Alabama at Birmingham
}

\subsection{6/lupus-2018-Ism.58}

Background Achieving remission or low lupus disease activity state (LDAS) in systemic lupus erythematosus (SLE) patients improves their prognosis in terms of damage accrual. But, there is not enough information about their impact on healthrelated quality of life (HRQoL). The aim of these analyses is to evaluate the association between remission or LDAS and HRQoL, after adjustment for possible confounders.

Methods The Almenara Lupus Cohort was started in 2012; all patients evaluated at the Rheumatology Department were invited to participate. Visits were performed every six months. Socioeconomic and clinical data were recorded at every visit. Disease activity was ascertained with the Systemic Lupus Erythematosus Disease Activity Index 2000 (SLEDAI-2K), disease damage with the SLICC/ACR Damage Index (SDI) and HRQoL was measured with the LupusQoL. For theses analyses, data from the baseline visit were included. Remission was defined as a SLEDAI-2K=0, prednisone $\leq 5 \mathrm{mg} / \mathrm{d}$, immunosuppressants on maintenance dose, LDAS was defined as not on remission and a SLEDAI- $2 \mathrm{~K} \leq 4$, prednisone $\leq 7.5 \mathrm{mg} / \mathrm{d}$, immunosuppressants on maintenance dose. Univariable and multivariable lineal regression models, adjusted by age at diagnosis, disease duration, socioeconomic status, antimalarial use, disease duration, time of exposure to prednisone, damage and comorbidities were performed. Due to the relatively small number of patients on remission, remission and LDAS were analyzed together.

Results Two hundred and eighty patients were included, 259 $(92.5 \%)$ were female, mean age at diagnosis was 35.4 $(\mathrm{SD}=13.5)$ years, disease duration was $7.2(6.5)$ years. Fortyfive $(16.1 \%)$ were on remission, 94 (33.6\%) were on LDAS. Being on remission or LDAS was associated with a better HRQoL in the following domains, independently of possible confounders: physical health, planning, emotional health and body image. Univariable and multivariable analyses are depicted in table 1 .
Conclusions Being on remission or LDAS is associated with a better HRQoL independently of possible confounders.

\begin{tabular}{|c|c|c|c|c|}
\hline & Univariable & & Multivariable & \\
\hline & B (C195\%) & $p$ value & B (C195\%) & $p$ value \\
\hline Physical Health & $8.68(3.01 ; 14.34)$ & 0.003 & $6.80(1.78 ; 11.82)$ & 0.008 \\
\hline Emotional Health & $6.22(0.71 ; 11.72)$ & 0.027 & $5.76(0.33 ; 11.19)$ & 0.038 \\
\hline Body image & $9.84(4.52 ; 15.17)$ & $<0.001$ & $9.31(4.03 ; 14.58)$ & 0.001 \\
\hline Pain & $6.74(0.83 ; 12.64)$ & 0.025 & $5.49(-0.10 ; 11.09)$ & 0.054 \\
\hline Planning & $9.70(2.91 ; 16.50)$ & 0.005 & $7.89(1.33 ; 14.42)$ & 0.018 \\
\hline Fatigue & $3.05(-3.15 ; 9.26)$ & 0.335 & $2.57(-3.55 ; 8.69)$ & 0.410 \\
\hline Intimate relationship & $6.09(-2.94 ; 15.13)$ & 0.186 & $5.11(-3.13 ; 13.36)$ & 0.224 \\
\hline Burden to others & $6.91(-0.18 ; 14.00)$ & 0.056 & $6.13(-0.91 ; 13.16)$ & 0.088 \\
\hline
\end{tabular}

\section{CS-24 ASSOCIATION OF LIPOPROTEIN SUBFRACTIONS AND GLYCOPROTEIN ACETYLATION WITH CORONARY PLAQUE BURDEN IN SYSTEMIC LUPUS ERYTHEMATOSUS}

Monica M Purmalek, Philip M Carlucci, Amit K Dey, Maureen Sampson, Yenealem Temesgen-Oyelakin, Simantini Sakhardande, Joseph B Lerman, Alice Fike, Michael Davis, Jonathan H Chung, Taufiq Salahuddin, Zerai Manna, Sarthak Gupta, Marcus Y Chen, Sarfaraz Hasni, Nehal N Mehta, Alan T Remaley, Mariana J Kaplan*. National Institute of Arthritis and Musculoskeletal and Skin Diseases (NIAMS) and National Heart, Lung and Blood Institute (NHLBI), National Institutes of Health, Bethesda, MD

\subsection{6/lupus-2018-Ism.59}

Background Subjects with Systemic Lupus Erythematosus (SLE) display an increased risk of atherosclerotic cardiovascular disease (CVD) that is not explained by Framingham Risk. This study sought to investigate the utility of nuclear magnetic resonance (NMR) spectroscopy measurements of serum lipoprotein particle counts and size and glycoprotein acetylation (GlycA) to predict coronary atherosclerosis in SLE.

Methods Coronary plaque burden was assessed in SLE subjects and healthy controls using coronary CT angiography. Lipoproteins and GlycA were quantified by NMR spectroscopy.

Results SLE subjects displayed statistically significant decreases in high-density lipoprotein (HDL) particle counts and increased very low density lipoprotein (VLDL) particle counts compared to controls. Non-calcified coronary plaque burden (NCB) negatively associated with HDL subsets, whereas it positively associated with VLDL particle counts in multivariate adjusted models. GlycA was significantly increased in SLE sera compared to controls. In contrast to high-sensitivity C-reactive protein, elevations in GlycA in SLE significantly associated with NCB and insulin resistance.

Conclusions SLE patients display a proatherogenic lipoprotein profile that may significantly contribute to the development of premature CVD. The results demonstrate that NMR measures of GlycA and lipoprotein profiles, beyond what is captured in routine clinical laboratory tests for lipids, could be a useful tool in assessing CVD risk in SLE patients. 